\title{
Investigation of restricted baby Skyrme models
}

\author{
C. Adam $^{a) *}{ }^{*}$ T. Romańczukiewicz ${ }^{b) \dagger}$ J. Sánchez-Guillén ${ }^{a) c) \ddagger}$ \\ A. Wereszczyński ${ }^{b) \S}$ \\ a) Departamento de Física de Partículas, Universidad \\ de Santiago, and Instituto Galego de Física de Altas Enerxías \\ (IGFAE) E-15782 Santiago de Compostela, Spain \\ b) Institute of Physics, Jagiellonian University, \\ Reymonta 4, Kraków, Poland \\ c) Sabbatical leave at: Departamento de Física Teórica, \\ Universidad de Zaragoza, 50009 Zaragoza, Spain
}

November 12, 2018

\begin{abstract}
A restriction of the baby Skyrme model consisting of the quartic and potential terms only is investigated in detail for a wide range of potentials. Further, its properties are compared with those of the corresponding full baby Skyrme models. We find that topological (charge) as well as geometrical (nucleus/shell shape) features of baby skyrmions are captured already by the soliton solutions of the restricted model. Further, we find a coincidence between the compact or non-compact nature of solitons in the restricted model, on the one hand, and the existence or nonexistence of multi-skyrmions in the full baby Skyrme model, on the other hand.
\end{abstract}

\footnotetext{
*adam@fpaxp1.usc.es

${ }^{\dagger}$ romanczukiewicz@th.if.uj.edu.pl

‡joaquin@fpaxp1.usc.es

§wereszczynski@th.if.uj.edu.pl
} 


\section{Introduction}

The baby Skyrme model was introduced originally as a planar analogue of the threedimensional Skyrme model [1]. Also its target space is simplified accordingly $\left(S^{2}\right.$ instead of the SU(2) target space of the Skyrme model) such that static field configurations can be classified according to their winding number in both cases. Like the Skyrme model, also the baby Skyrme model consists of a quadratic kinetic term (the $\mathrm{O}(3)$ nonlinear sigma model term), a quartic kinetic term (the analogue of the Skyrme term), and a potential. For the baby Skyrme model, the inclusion of a potential term is obligatory for the existence of static finite energy solutions. The specific form of this potential term is, however, quite arbitrary, and different potentials have been studied [2] - [5] (for some recent studies see e.g. [6], [7]). In addition to its role as a toy model for the Skyrme model, the baby Skyrme model has also found some independent applications in condensed matter physics in the description of the quantum Hall effect [8].

The energy functional of the baby Skyrme model for static configurations is

$$
E=\frac{1}{2} \int d^{2} x\left(\partial_{i} \vec{\phi} \cdot \partial_{i} \vec{\phi}+\frac{1}{4}\left(\epsilon_{i j} \partial_{i} \vec{\phi} \times \partial_{j} \vec{\phi}\right)^{2}+\mu^{2} V(\vec{\phi})\right)
$$

where $\vec{\phi}$ is a three-component vector field with unit modulus $|\vec{\phi}|=1$, and $V$ is the potential. Further, $\mu$ is a positive constant.

Although the baby Skyrme model, due to its simpler structure, may offer a way for a better understanding of the solutions in the $(3+1)$ Skyrme model, it is still a non-integrable, highly complicated, topologically non-trivial, non-linear field theory. Many properties of baby skyrmions are established mainly by numerical simulations, whereas it is much more difficult to gain analytical understanding. Therefore, it is important to know whether there exists the possibility for any further simplification which would keep us in the class of Skyrme-like models and, nevertheless, allows for some exact analytical calculations. For example, one may try to identify which features of the solutions of the baby Skyrme model are governed by which part of the model. Then, neglecting a particular part of the Lagrangian, one could investigate a simplified model. In case of static solutions only two simplifications are possible. One may either suppress both the potential and the quartic, pure Skyrme term, which leads to the scale invariant $\mathrm{O}(3)$ sigma model with its well-known meromorphic solutions. Or one may opt for an energy functional which is not scale invariant. In this case, both the quartic kinetic term and the potential are mandatory for the evasion of Derrick's theorem, whereas the quadratic term is optional from this point of view. The latter fact led Gisiger and Paranjape to consider the model with the quadratic term omitted [9], and with the so-called "old" potential

$$
V(\vec{\phi})=(\hat{n}-\vec{\phi})^{2}=2(1-\hat{n} \cdot \vec{\phi})
$$

where $\hat{n}$ is a constant unit vector which selects the vacuum. The resulting model has a huge amount of symmetry. Indeed, both the area-preserving diffeomorphisms on base space [9] and an abelian subgroup of the area-preserving difeomorphisms on target space [10] are symmetries of the static GP (=Gisiger and Paranjape) model (as the model consists of a potential term and the pure Skyrme term only, we will also call it the "pure baby Skyrme model"). Further, already for a spherically symmetric ansatz, GP found an infinite number of exact soliton solutions, which, together with the huge symmetry, points towards the integrability of this model in $(2+0)$ dimensions [11]. 
These solitons are, in fact, compact, that is, they differ from the vacuum only in a finite compact region of the (two-dimensional) base space [12]. A further explanation of their compact nature can be found in [7], where it is demonstrated that the GP model can, in fact, be mapped to the signum-Gordon model (the latter model is known to support compact solutions, see [13]). In addition, GP derived a Bogomolny bound in their model, but the solutions they found do not saturate this bound.

The main aim of the present work is to understand correlations between properties of soliton solutions in the pure and the full baby Skyrme models. It occurs that some important topological as well as geometrical features of baby skyrmions can be captured already by solutions of the simplified model.

First, we show that the previously proven integrability of the static version of the pure baby Skyrme models [11] can be extended to the $(2+1)$ space-time case. However, now the models are integrable in the sense of generalized integrability [14]. In particular, we construct an infinite set of conserved quantities.

In a next step, we generalize the results of GP by studying pure baby Skyrme models with different potentials. We find that, depending on the type of potential we choose, there exist soliton solutions with qualitatively different behavior. In some cases, there exist compact solitons, whereas in other cases the solitons have an exponential tail or are even only localized by some inverse powers of the radius. Further, we find an interesting coincidence between the behavior of the solitons in the GP like model (with only a quartic kinetic term), on the one hand, and the existence of multi-soliton solutions of the full baby Skyrme model, on the other hand. Multi-solitons in the full model seem to exist only for potentials such that the GP model has compacton solutions, whereas they do not exist if the solitons of the corresponding GP model have an exponential or power-like tail. Moreover, also the nucleus respectively shell-like structure is preserved when we go from the pure to the full baby Skyrme model.

We also comment on the issue of Bogomolny bounds. Specifically, we point out that there exists a second Bogomolny bound in the GP model, and all exact soliton solutions saturate this bound. Further, this bound continues to hold for GP like models with arbitrary potential. This bound was originally found in [15], as a contribution to an improved Bogomolny bound for the full baby Skyrme model. We just add the information that in GP like models this bound is, in fact, saturated, and solutions are, therefore, solutions of the corresponding Bogomolny equations. Further, this second Bogomolny bound of the GP model immediately leads to a second, improved Bogomolny bound for the full baby Skyrme model [15]. Solutions of the full baby Skyrme model do not saturate this bound for general potentials. This second bound is, however, much tighter than the more widely known Bogomolny bound which stems from the quadratic $\mathrm{O}(3)$ sigma model term alone. In addition, the new bound becomes very tight in the limits of very weak or very strong potential. We explore the quality (i.e. tightness) of the bound for several parameter choices. We remark that an equivalent Bogomolny bound may be derived for generalized Skyrme models in three dimensions [17]. There the pertinent topological charge is the baryon number, and the limiting model which saturates the Bogomolny bound gives an accurate description of basic properties of nuclei, like e.g. masses and sizes.

Finally, we comment on time-dependent, non-topological solutions in the pure baby Skyrme model. 


\section{Generalized integrability in (2+1) dimensions}

The model we are going to investigate in this work is defined by the following Lagrange density

$$
L=\frac{1}{2}\left(\frac{1}{2}\left(\partial_{\mu} \vec{\phi} \times \partial_{\nu} \vec{\phi}\right)^{2}+\mu^{2} V\left(\phi^{3}\right),\right)
$$

where the potential $V$ is any potential depending only on the third component of the unit vector field. In applications to the quantum Hall effect, this restricted model corresponds to the limit of infinitely strong magnetic field. After using the standard stereographic projection

$$
\vec{\phi}=\frac{1}{1+|u|^{2}}\left(u+\bar{u},-i(u-\bar{u}), 1-|u|^{2}\right)
$$

we derive equation of motion

$$
\partial_{\mu} \mathcal{K}^{\mu}-\frac{\mu^{2}}{4} \bar{u}\left(1+|u|^{2}\right)^{2} V^{\prime}=0
$$

where $\left(u_{\mu} \equiv \partial_{\mu} u\right.$, etc. $)$

$$
\mathcal{K}^{\mu}=\frac{K^{\mu}}{\left(1+|u|^{2}\right)^{2}}, \quad K^{\mu}=\left(u_{\nu} \bar{u}^{\nu}\right) \bar{u}^{\mu}-\bar{u}_{\nu}^{2} u^{\mu}
$$

and the prime denotes differentiation with respect to $u \bar{u}$. The construction of conserved quantities is performed by a well understood procedure originally described in [14]. The conserved currents are chosen as

$$
J_{\mu}=\frac{\delta G}{\delta \bar{u}} \mathcal{K}_{\mu}-\frac{\delta G}{\delta u} \overline{\mathcal{K}}_{\mu}, \quad G=G(u \bar{u}),
$$

where $G$ is an arbitrary function which depends on the modulus of the complex field. Obviously, we have infinitely many such currents as these functions are not restricted to any particular form. Then, one may easily calculate the divergence of the currents and get

$\partial_{\mu} J^{\mu}=\frac{\delta^{2} G}{\delta \bar{u}^{2}} \mathcal{K}_{\mu} \bar{u}^{\mu}+\frac{\delta^{2} G}{\delta \bar{u} \delta u} \mathcal{K}_{\mu} u^{\mu}+\frac{\delta G}{\delta \bar{u}} \partial^{\mu} \mathcal{K}_{\mu}-\frac{\delta^{2} G}{\delta u^{2}} \overline{\mathcal{K}}_{\mu} u^{\mu}-\frac{\delta G}{\delta u \delta \bar{u}} \overline{\mathcal{K}}_{\mu} \bar{u}^{\mu}-\frac{\delta G}{\delta u} \partial^{\mu} \overline{\mathcal{K}}_{\mu}$.

Using the properties of $\mathcal{K}_{\mu}$

$$
\mathcal{K}_{\mu} \bar{u}^{\mu}=\overline{\mathcal{K}}_{\mu} u^{\mu}, \quad \mathcal{K}_{\mu} u^{\mu}=\overline{\mathcal{K}}_{\mu} \bar{u}^{\mu}
$$

we find

$$
\partial_{\mu} J^{\mu}=G^{\prime}\left(u \partial^{\mu} \mathcal{K}_{\mu}-\bar{u} \partial^{\mu} \overline{\mathcal{K}}_{\mu}\right)=0,
$$

where the last step follows from the equation of motion.

In contrast to the pure baby Skyrme model, its full version is not integrable, resulting in a rather complicated dynamics of the baby skyrmions. It has been shown, however, that the full baby Skyrme model possesses an integrable submodel defined by an additional constraint which must be imposed in addition to the equations of motion, namely the eikonal equation $\left(\partial_{\mu} u\right)^{2}=0$. This constraint is quite restrictive and it is not known wether this integrable submodel can lead to interesting solutions. On the contrary, the pure baby Skyrme model is integrable (in the specific meaning of the generalized 
integrability) without any additional constraint.

One may notice, that the pure baby Skyrme model explores an integrable limit of the full baby model as was originally considered by Aratyn et.al. in the case of the SkyrmeFaddeev-Niemi model [18]. Indeed, their limit $m^{2} \rightarrow 0$ leads to a model without the quadratic part of the action. However, as they omitted the potential term, as well, the limit gave a theory without static soliton solutions, which probably was the reason why they did not investigate that model in detail.

\section{Solitons in the pure baby Skyrme model}

\subsection{One-vacuum potentials}

Let us consider the energy functional for the pure baby Skyrme model

$$
E=\frac{1}{2} \int d^{2} x\left(\frac{1}{4}\left(\epsilon_{i j} \partial_{i} \vec{\phi} \times \partial_{j} \vec{\phi}\right)^{2}+\mu^{2} V\left(\phi^{3}\right)\right),
$$

where we specify the potential as

$$
V=4\left(\frac{1-\phi^{3}}{2}\right)^{s}
$$

which for $s=1$ gives the old baby Skyrme potential. Here $s>0$. Such a family of generalized old baby potentials has been analyzed by Karliner and Hen in the context of the rotational symmetry breaking in the full baby Skyrme model [6].

After the stereographic projection we get

$$
E=\int d^{2} x\left(2 \frac{(\nabla u \nabla \bar{u})^{2}-(\nabla u)^{2}(\nabla \bar{u})^{2}}{\left(1+|u|^{2}\right)^{4}}+2 \mu^{2}\left(\frac{|u|^{2}}{1+|u|^{2}}\right)^{s}\right),
$$

Further, we assume the symmetric ansatz

$$
u=e^{i n \varphi} f(r)
$$

which is compatible with the equations of motion, and introduce a new function

$$
1-g=\frac{1}{1+f^{2}}
$$

together with a new variable $y=r^{2} / 2$. For topologically non-trivial solution one has to impose the following boundary conditions

$$
f(r=0)=\infty, \quad f(r=R)=0, \quad f^{\prime}(r=R)=0
$$

where $R$ is finite for compactons or $R=\infty$ for standard solitons (then, the third expression does not lead to a new condition). In terms of the new function $g$ we get

$$
g(y=0)=1, \quad g(y=Y)=0, \quad g^{\prime}(y=Y)=0,
$$

Now, the energy takes the simply form

$$
E=2 \pi \int d y\left(2 n^{2} g_{y}^{2}+2 \mu^{2} g^{s}\right) .
$$


The finite energy solutions must obey the pertinent first order Bogomolny equation which is easily obtained from the second order equation of motion,

$$
n^{2} g_{y}^{2}=\mu^{2} g^{s} .
$$

This simple equation supports topological solutions (i.e., solutions with the previously specified boundary conditions), which may be divided into three classes.

(i) $s \in(0,2)$ - Compactons

The pertinent solutions are compactons

$$
g(y)=\left\{\begin{array}{cc}
\left(1-\frac{y}{y_{0}}\right)^{\frac{2}{2-s}} & 0 \leq y \leq y_{0} \\
0 & y \geq y_{0}
\end{array}\right.
$$

where the boundary of the compacton is located at

$$
y_{0}=\frac{2 n}{\mu(2-s)} .
$$

The approach to the vacuum becomes more and more rapid as we tend to $s=2$. Such compact solutions represent rotationally symmetric multi-solitons configuration which can be understood as a collection of $n Q=1$ solitons located on top of each other. However, due to the compacton nature of the solutions one may easily construct a nonrotational solution with $Q=n$ by a collection of some solutions with lower topological charges, provided that they are sufficiently separated and the sum of charges is equal to $n$.

(ii) $s=2$ - Exponentially localized solitons

Now, we get a soliton with the standard exponential tails

$$
g(y)=e^{-\frac{\mu y}{n}} .
$$

Again, higher charge solitons may be interpreted as several $Q=1$ solitons sitting on top of each other. Further, it is no longer obvious how to construct non-symmetric solutions and solutions corresponding to well-separated solitons. We remark that this case has already been investigated in [16].

(iii) $s>2$ - Power-like localized solitons

The third class of solutions is formed by solitons with power-like tails

$$
g(y)=\left(\frac{y_{0}}{y_{0}+y}\right)^{\frac{2}{s-2}},
$$

where

$$
y_{0}=\frac{2 n}{\mu(s-2)}
$$

Three remarks are appropriate.

Firstly, as one could expect, in the case of solutions of a first order Bogomolny equation, for all models the total energy is proportional to the topological charge

$$
E=\frac{16 \pi}{s+2} \mu|Q|
$$

Secondly, from a geometrical point of view all these solutions share a common feature. Namely, they are of nucleus type with their energy density concentrated around the 
origin. This resembles the baby skyrmions in the old baby model, where solitons also possess such a nucleus shape.

Finally, there is a very intriguing coincidence between the compactness of soliton solutions of the pure model and the existence, for some values of the parameters, of multi baby-skyrmions in the full model. In fact, for potentials with $s \in(0,2)$ solitons in the pure model are of the compact type. On the other hand, as is known from previous numerical works, full baby Skyrme models with the same potentials support multi soliton solutions. Moreover, for baby Skyrme models with potentials with $s \geq 2$, no multi-soliton configurations have been found. It corresponds, on the pure model side, to non-compact topological solutions (exponentially or power-like localized). It seems as if the instability of the multi-skyrmions in the full baby models has its origin in the non-compactness of the solitons in the pure models. Equivalently, one may conjecture that compactons in the pure models are seeds for multi-skyrmions in the full theory.

\subsection{Two-vacua potentials}

\subsubsection{Generalized new baby potential}

Another type of potentials we are going to analyze is given by the expression

$$
V=4\left(\frac{1-\left(\phi^{3}\right)^{2}}{4}\right)^{s},
$$

with $s>0$. In the limit $s=1$ one can recognize the new baby potential, a well-known potential with two vacua $\phi^{3}= \pm 1$. The pertinent first order equation of motion reads

$$
n^{2} g_{y}^{2}=\mu^{2}(g(1-g))^{s} .
$$

For this potential we find two classes of solutions.

(i) $s \in(0,2)$ - Compactons

The general solution describes a compact ring. In fact, both vacua are of the compacton type i.e., a solution tends to them in a power like manner. More precisely, the solution (not in explicit form) reads

$$
\frac{2}{2-s_{2}} F_{1}\left[1-\frac{s}{2}, \frac{s}{2}, 2-\frac{s}{2}, g\right]=\frac{\mu\left(y+y_{0}\right)}{n},
$$

where ${ }_{2} F_{1}$ is a hypergeometric function. An example of an explicit solution can be found for $s=1$

$$
g(y)=\left\{\begin{array}{cc}
\left(\sin \frac{2 \mu\left(y-y_{0}\right)}{n}\right)^{2} & 0 \leq y \leq y_{0} \\
0 & y \geq y_{0}
\end{array}\right.
$$

and

$$
y_{0}=\frac{n \pi}{4 \mu} .
$$

The corresponding energy density is not concentrated at the origin but has its maximum at $y_{\max }=n \pi / 8 \mu$, forming a ring-like structure. This solution can be easily extended to a family of ring-like solutions with the maximum of the energy density located at any $y \geq y_{\max }$. Again, all solutions lead to a linear energy-charge relation.

Let us underline that the fact that solutions are of the ring type again resembles the geometric properties of soliton solutions in the full baby Skyrme model. Indeed, the 
baby skyrmions in the new baby Skyrme model have this property.

(ii) $s \geq 2$ - No solitons

It is easy to check that there are no topological solitons for such a value of the parameter. The reason for this is that both vacua are approached exponentially $(s=2)$ or polynomially $(s>2)$. It is of course impossible to join both vacua in such a way on the semi-infinite segment $y \in[0, \infty)$.

\subsubsection{Generalized new baby potential - non-symmetric case}

The last family of potentials of this type we want to analyze has the form

$$
V=4\left(\frac{1+\phi^{3}}{2}\right)^{a}\left(\frac{1-\phi^{3}}{2}\right)^{b},
$$

with $a, b>0$. The properties of the solutions are as follows.

(i) $a \in(0,2)$ and $b \in(0,2)$ - Compactons

We get compactons as the approach to both vacua is in a power-like manner.

(ii) $a=2$ and $b \in(0,2)$ or $a \in(0,2)$ and $b=2$ - Exponentially localized solitons

We get standard soliton solutions with exponential tails. As an example, we get

$$
g(y)=\frac{2}{1+\cosh \frac{\mu y}{n}}
$$

for the first case $(a=2, b=1)$, and

$$
g(y)=\left(\tanh \frac{\mu y}{2 n}\right)^{2},
$$

for second case ( $a=1, b=2)$, respectively. Notice that the second solution possesses the reversed boundary conditions, i.e., $g(0)=0, g(\infty)=1$.

(iii) $a>2$ and $b \in(0,2)$ or $a \in(0,2)$ and $b>2$ - Power-like localized solitons

We get solitons with power-like tails. As an example one may consider the choice of parameters $a=4, b=1$. Then, the pertinent solution reads

$$
\sqrt{\frac{1}{g^{2}}-\frac{1}{g}}+\operatorname{arcosh} \frac{1}{\sqrt{g}}=y .
$$

All presented solutions can be extended to shell-like configurations. As before one gets that the energy is proportional to the topological charge.

(iii) $a \geq 2$ and $b \geq 2$ - No solitons

\subsection{Vortex potentials}

A different family of potentials also considered for the baby Skyrme model is given by the following expression

$$
V=4\left(\left(\phi^{3}\right)^{2}\right)^{s}\left(\frac{1-\phi^{3}}{2}\right),
$$

where $s>0$. Here the vacuum manifold is completely different from the cases considered up to now. It still consists of two disconnected components, but now only one of the two components is a point (the north pole $\phi^{3}=1$ of the target space $S^{2}$ ), whereas the other component of the vacuum is a circle $S^{1}: \phi^{3}=0,\left(\phi^{1}\right)^{2}+\left(\phi^{2}\right)^{2}=1$. In 
our parametrization, this vacuum corresponds to $g=1 / 2$. The presence of this new vacuum allows for field configurations which approach it in the limit $|\vec{x}| \rightarrow \infty$. Such fields may be divided into disjoint topological classes as

$$
\vec{n}_{\infty}: \mathbb{R}_{r \rightarrow \infty}^{2} \simeq \mathbb{S}^{1} \longrightarrow U(1) \simeq \mathbb{S}^{1}
$$

The relevant topological index is the winding number $Q \in \pi_{1}\left(S^{1}\right)$, and the corresponding field configurations are vortices. The important question is, of course, whether such vortex configurations are possible solutions of the underlying field theory. In this respect, the full and the pure baby Skyrme model are fundamentally different. The reason is that the quadratic $\mathrm{O}(3)$ sigma model term, which is present only in the full model, produces infinite energy for vortex configurations. Indeed, in polar coordinates the static $\mathrm{O}(3)$ term essentially is the sum of a radial gradient squared and an angular gradient squared. In the limit, the angular gradient behaves like $r^{-1} \hat{e}_{\varphi} \partial_{\varphi} \exp (i n \varphi)=i n r^{-1} \hat{e}_{\varphi}$ where $n$ is the vortex number, so for nonzero $n$ the energy density goes like $r^{-2}$ and produces a logarithmic divergency. As a consequence, there are no vortices with finite energy in the full baby Skyrme model. In the pure model, on the other hand, only the quartic term is present in addition to the potential. Further, due to its antisymmetry, both gradients have to appear in this term (in fact, both quadratically). Therefore, the slowly decaying angular gradient is always multiplied by a rapidly decaying radial gradient, such that the resulting energy density decays sufficiently fast to have finite energy. In short, finite energy vortices are not excluded in the pure model, and they turn out to exist, as we shall see in a moment.

(i) $s \in(0,1)$ - Vortex compactons

We get compact vortices. As an example with explicit solutions let us consider the model with $s=1 / 2$. Then,

$$
g(y)=\left\{\begin{array}{cc}
\frac{1}{2}\left(\cosh \frac{4 \sqrt{2}\left(y-y_{0}\right)}{n}\right)^{2} & y \leq y_{0} \\
\frac{1}{2} & y>y_{0},
\end{array}\right.
$$

or

$$
g(y)=\left\{\begin{array}{cc}
\frac{1}{2}\left(\sin \frac{4 \sqrt{2} y}{n}\right)^{2} & y \leq \tilde{y}_{0} \\
\frac{1}{2} & y \geq \tilde{y}_{0},
\end{array}\right.
$$

where

$$
y_{0}=\frac{n}{4 \sqrt{2}} \operatorname{arccosh} \sqrt{2}, \quad \tilde{y}_{0}=\frac{\pi n}{8 \sqrt{2}} .
$$

The first solution represents vortex $(g \in(1,1 / 2))$ whereas the second anti-vortex $(g \in(0,1 / 2))$. Quite interestingly, because of the compactness of the solutions one may construct a configuration carrying a non-trivial value of the topological charge by gluing in a proper way together both profile functions (i.e., both vortices). Namely,

$$
g(y)=\left\{\begin{array}{cc}
\frac{1}{2}\left(\cosh \frac{4 \sqrt{2}\left(y-y_{<}\right)}{n}\right)^{2} & y \in\left[0, y_{<}\right] \\
\frac{1}{2} & y \in\left[y_{<}, y_{<}+y_{0}\right] \\
\frac{1}{2}\left(\sin \frac{4 \sqrt{2}\left(y-\left(y_{>}+y_{0}\right)\right)}{n}\right)^{2} & y \in\left[y_{<}+y_{0}, y_{>}+y_{0}\right] \\
0 & y>y_{>}+y_{0},
\end{array}\right.
$$


where

$$
\begin{gathered}
y_{<}=\frac{n}{4 \sqrt{2}} \operatorname{arccosh} \sqrt{2} \\
y_{>}=\frac{\pi n}{8 \sqrt{2}}\left(1+\frac{2}{\pi} \operatorname{arccosh} \sqrt{2}\right) .
\end{gathered}
$$

Thus, two vortices with the same winding numbers $n$ form a baby skyrmion with the topological charge $Q=n$. Therefore, for such potentials a baby skyrmion seems to dissolve into a pair of $U(1)$ vortices, which now are the true constituent topological objects.

(ii) $s \geq 1$ - Exponential or polynomial vortices

A pertinent example is given by $s=1$ case. Then,

$$
g(y)=\frac{1}{2}\left(\operatorname{coth} \frac{2\left(y+y_{0}\right)}{n}\right)^{2}
$$

or

$$
g(y)=\frac{1}{2}\left(\tanh \frac{2 y}{n}\right)^{2}
$$

where $y_{0}$ obeys

$$
\operatorname{coth} \frac{2 y_{0}}{n}=\sqrt{2} \text {. }
$$

Of course, now these vortex solutions cannot be glued together to form a stable configuration with non-zero topological charge.

\section{Bogomolny bounds}

In this section we prove that the solutions of the previous section are indeed of the Bogomolny type, without performing any dimensional reduction. Moreover, we derive the pertinent Bogomolny bound and show that our solutions saturate it. In fact, in an investigation of the full baby Skyrme model this bound has been originally reported in [15]. Let us begin by reviewing briefly the original Bogomolny bound found by GP. They studied the energy functional

$$
E=\frac{1}{2} \int d^{2} x\left(\frac{1}{4}\left(\epsilon_{i j} \partial_{i} \vec{\phi} \times \partial_{j} \vec{\phi}\right)^{2}+\mu^{2}(\hat{n}-\vec{\phi})^{2}\right)
$$

where $\hat{n}$ is a constant unit vector selecting the vacuum. Further, they derived the following Bogomolny bound

$$
\begin{aligned}
E & =\frac{1}{2} \int d^{2} x\left(\left(\frac{1}{2} \epsilon_{i j} \partial_{i} \vec{\phi} \times \partial_{j} \vec{\phi} \pm \mu(\hat{n}-\vec{\phi})\right)^{2} \mp \mu(\hat{n}-\vec{\phi}) \cdot \epsilon_{i j} \partial_{i} \vec{\phi} \times \partial_{j} \vec{\phi}\right) \\
& =\frac{1}{2} \int d^{2} x\left(\left(\frac{1}{2} \epsilon_{i j} \partial_{i} \vec{\phi} \times \partial_{j} \vec{\phi} \pm \mu(\hat{n}-\vec{\phi})\right)^{2} \pm \mu \epsilon_{i j} \vec{\phi} \cdot\left(\partial_{i} \vec{\phi} \times \partial_{j} \vec{\phi}\right)\right)
\end{aligned}
$$

where a total divergence term which gives zero upon integration has been omitted in the last expression. The energy, therefore, obeys a Bogomolny bound

$$
E \geq E_{B} \equiv 4 \pi \mu|Q|
$$


were the integer-valued topological charge $Q$ is

$$
Q=\frac{1}{8 \pi} \int d^{2} x \epsilon_{i j} \vec{\phi} \cdot\left(\partial_{i} \vec{\phi} \times \partial_{j} \vec{\phi}\right)
$$

The solutions of GP (see also section 3.1), however, do not saturate this Bogomolny bound. Instead, they satisfy

$$
E_{\mathrm{sol}}=\frac{4}{3} E_{B}
$$

so they are still proportional to the topological charge, just the coefficient in front is slightly bigger than the coefficient for the Bogomolny bound. Firstly, let us remark that nontrivial solutions saturating this bound cannot exist. Indeed, the corresponding Bogomolny equation is

$$
\frac{1}{2} \epsilon_{i j} \partial_{i} \vec{\phi} \times \partial_{j} \vec{\phi} \pm \mu(\hat{n}-\vec{\phi})=0
$$

and multiplying this by $\times \vec{\phi}$ one easily concludes

$$
\hat{n} \times \vec{\phi}=0
$$

and any solution $\vec{\phi}$ must be proportional to the trivial constant vacuum vector $\hat{n}$.

Secondly, the fact that the explicit solutions still are linear in the topological charge, just with a slightly bigger prefactor, already indicates that there might exist another, tighter Bogomolny bound which is saturated by the energies of the solutions. We will demonstrate now that this is indeed the case (the bound itself has already been found in [15], as a contribution to an improved bound for the full baby Skyrme model). For this purpose, we observe first that the vectorial expression whose square gives the quartic kinetic term necessarily is proportional to the vector $\vec{\phi}$ itself and may therefore be written like

$$
\epsilon_{i j} \partial_{i} \vec{\phi} \times \partial_{j} \vec{\phi}=\vec{\phi}\left(\vec{\phi} \cdot \epsilon_{i j} \partial_{i} \vec{\phi} \times \partial_{j} \vec{\phi}\right) .
$$

Further, we choose the vacuum vector $\hat{n}=(0,0,1)$ such that the potential $V=2(1-$ $\left.\phi^{3}\right)$ depends only on $\phi^{3}$. We will, in fact, allow for general potentials $V=V\left(\phi^{3}\right)$ at the moment, because the Bogomolny bound can be easily found for them. Using this, we rewrite the energy functional like follows,

$$
\begin{gathered}
E=\frac{1}{2} \int d^{2} x\left(\frac{1}{4}\left(\epsilon_{i j} \vec{\phi} \cdot\left(\partial_{i} \vec{\phi} \times \partial_{j} \vec{\phi}\right)\right)^{2}+\mu^{2} V\left(\phi^{3}\right)\right) \\
=\frac{1}{2} \int d^{2} x\left(\left(\frac{1}{2} \epsilon_{i j} \vec{\phi} \cdot\left(\partial_{i} \vec{\phi} \times \partial_{j} \vec{\phi}\right) \pm \mu \sqrt{V}\right)^{2}\right. \\
\left.\mp \mu \sqrt{V} \epsilon_{i j} \vec{\phi} \cdot\left(\partial_{i} \vec{\phi} \times \partial_{j} \vec{\phi}\right)\right) .
\end{gathered}
$$

It remains to demonstrate that the last term above is, indeed, a Bogomolny energy which is bounded by the topological charge. For this purpose we use the complex field $u$ together with its modulus and phase $u=f e^{i \Sigma} \equiv \sqrt{F} e^{i \Sigma}$ instead of the unit vector field $\vec{\phi}$. In these terms the topological charge reads

$$
Q[u]=\frac{-1}{2 \pi i} \int d^{2} x \frac{\epsilon_{i j} u_{i} \bar{u}_{j}}{(1+u \bar{u})^{2}}=\frac{1}{2 \pi} \int d^{2} x \epsilon_{i j} \frac{F_{i} \Sigma_{j}}{(1+F)^{2}} .
$$


For the energy we find from (24)

$$
E \geq \mp 4 \pi \mu \int d^{2} x\left[\frac{1}{2 \pi} \sqrt{V(F)} \epsilon_{i j} \frac{F_{i} \Sigma_{j}}{(1+F)^{2}}\right] \equiv 4 \pi \mu C_{1}|Q|
$$

where the sign has to be chosen accordingly (upper sign for $Q>0$ ). Further, $C_{1}$ is a constant which depends on $V$, and the last equality still has to be proven. If we replace $V$ by one, then the result is obvious, because the integrand is just the integrand of the topological charge (25). Equivalently, this expression is just the pullback of the area form on the target space $S^{2}$, normalized to one. The base space integral gives the result $Q$ because the base space sphere is covered $Q$ times while the target space sphere is covered once. But this last result continues to hold with the factor $\sqrt{V(F)}$ present, up to a constant $C_{1}$. Indeed, we just have to find a new target space coordinate $\tilde{F}$ instead of $F$ such that

$$
\frac{\sqrt{V(F)} d F}{(1+F)^{2}}=C_{1} \frac{d \tilde{F}}{(1+\tilde{F})^{2}} .
$$

The constant $C_{1}$, and a second coordinate $C_{2}$ which is provided by the integration of Eq. (26), are needed to impose the two conditions

$$
\tilde{F}(F=0)=0, \quad \tilde{F}(F=\infty)=\infty
$$

which must hold if $\tilde{F}$ is a good coordinate on the target space $S^{2}$. Obviously, $C_{1}$ depends on the potential. Specifically, for the "old" potential

$$
V=2\left(1-\phi^{3}\right)=4 \frac{F}{1+F}
$$

we find

$$
\begin{gathered}
2 \sqrt{\frac{F}{1+F}} \frac{d F}{(1+F)^{2}}=C_{1} \frac{d \tilde{F}}{(1+\tilde{F})^{2}} \\
\frac{4}{3}\left(\frac{F}{1+F}\right)^{\frac{3}{2}}=-\frac{C_{1}}{1+\tilde{F}}+C_{2}
\end{gathered}
$$

and, finally, from the boundary conditions $(27), C_{1}=C_{2}=(4 / 3)$.

The corresponding Bogomolny equations are

$$
\frac{1}{2} \epsilon_{i j} \vec{\phi} \cdot\left(\partial_{i} \vec{\phi} \times \partial_{j} \vec{\phi}\right) \pm \mu \sqrt{V\left(\phi^{3}\right)}=0
$$

or, in terms of $u=f e^{i \Sigma}, F=f^{2}$,

$$
2 \epsilon_{i j}\left[\partial_{i}(1+F)^{-1}\right] \partial_{j} \Sigma \pm \mu \sqrt{V(F)}=0 .
$$

For $F=F(r), \Sigma=n \varphi$ this just gives the two possible square roots of the first order equations found in Section 3, as may be checked easily.

\subsection{Bogomolny bounds in the full baby Skyrme model}

Here we want to discuss the fact that the above Bogomolny bound may be used immediately to derive a tighter bound also for the full baby Skyrme model [15]. The important 
point here is that the quadratic, $\mathrm{O}(3)$ sigma model part of the full baby Skyrme model, which is absent in the GP model, has its own, independent Bogomolny bound

$$
E_{O(3)} \geq 4 \pi|Q|
$$

in terms of the same winding number. For the full baby Skyrme model (1) we get, therefore, in the case of the "old" potential, the improved Bogomolny bound

$$
E_{b S}=E_{O(3)}+E_{G P} \geq 4 \pi|Q|\left(1+\frac{4}{3} \mu\right) .
$$

In the full baby Skyrme model, however, solutions no longer saturate this bound, because they would have to obey the field equations both of the $\mathrm{O}(3)$ model and of the GP model in order to do so. Nevertheless, we expect that the bound should be very tight

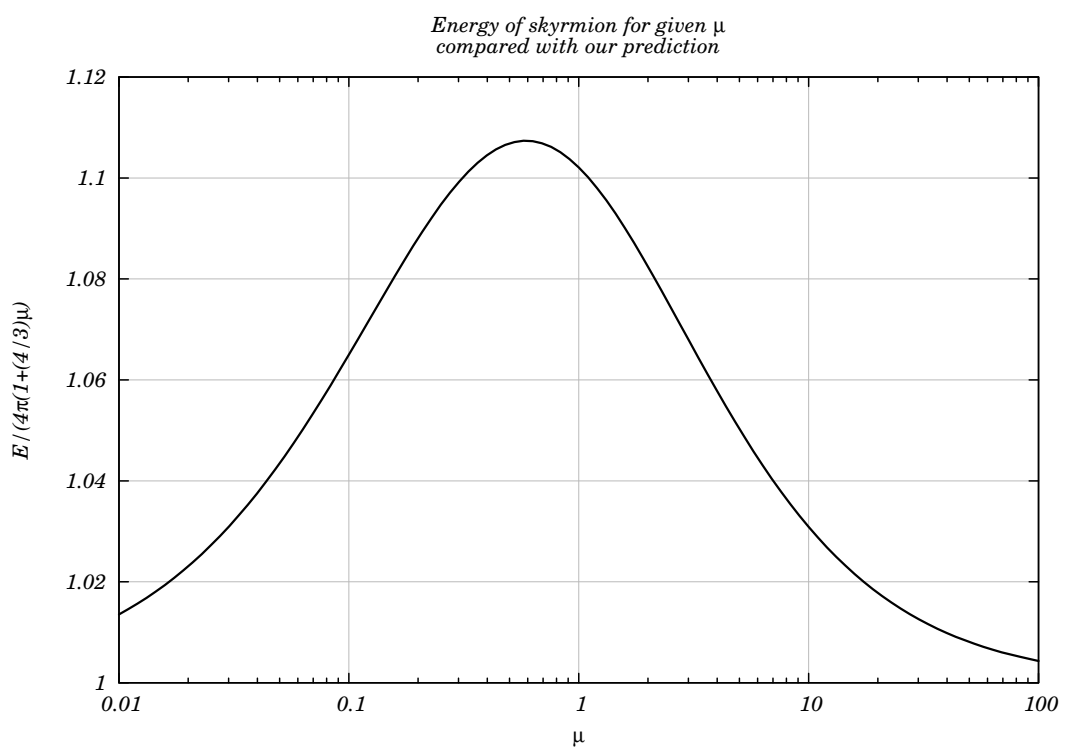

Figure 1: Comparison of the energy and Bogomolny bound for $Q=1$ baby skyrmion

for very small and very large $\mu$. The reason for this is as follows. A simple scaling argument shows that the potential and the quartic pure Skyrme term always contribute the same amount of energy to a solution. This implies that in the limit of very small $\mu$, the energy approaches the pure quadratic $\mathrm{O}(3)$ sigma model energy, whereas in the opposite limit of large $\mu$, the energy approaches the energy of the GP model. But both the pure $\mathrm{O}(3)$ model energy and the pure GP model energy saturate their respective Bogomolny bounds. A simple numerical calculation of the energies confirms this expectation. We make the rotationally symmetric ansatz $u=f(r) e^{i m \varphi}$ and determine $f(r)$ and the corresponding energy by a simple shooting algorithm. The deviation between energy and bound reaches its maximum of about $10 \%$ for values of $\mu \sim 1$, and diminishes both for small and large $\mu$, as expected, see Fig. 1. The case $\mu=\sqrt{0.1}$ and $Q=1$ corresponds to the numerical solution originally reported in the first reference of [2], $E=1.564 \times 4 \pi$, in which case the new bound gives $1.422 \times 4 \pi$ which is definitely better than the old pure $\mathrm{O}(3)$ sigma model bound $4 \pi$ (10\% off instead of more than $50 \%$ off). 


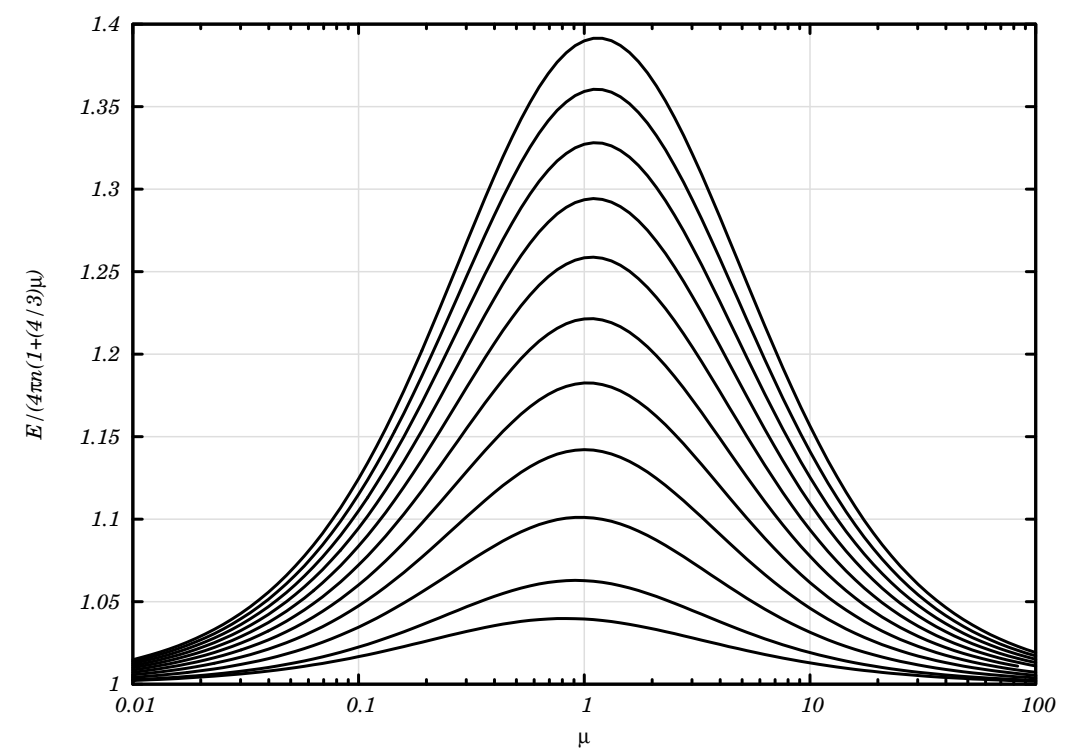

Figure 2: Comparison of the energy and Bogomolny bound for $Q=2 . .12$ baby skyrmions

In Fig. 2 we show the corresponding results for higher topological charges $Q=$ $2, \ldots, 12$. Observe that although the symmetric ansatz does not necessarily lead to the energy minima of the full baby Skyrme model, the bound is again very tight in the large and small $\mu$ limits.

The generalization to other potentials is obvious. The bound is always of the type

$$
E_{b S}=E_{O(3)}+E_{G P} \geq 4 \pi|Q|\left(1+C_{1} \mu\right)
$$

where the numerical factor $C_{1}$ depends on the specific potential but can be calculated exactly. We remark that the limit of large $\mu$ has already been studied in [16], although for a different potential (the square of the "old" potential).

\section{Non-existence of non-topological $Q$-balls}

Here we show that there are no topologically trivial $Q$-balls in the pure baby model with the old potential.

We assume the symmetric ansatz for time-dependent solutions

$$
u=e^{i(\omega t+n \phi)} f(r) .
$$

Then, the equation of motion takes the form

$$
f\left[-\frac{1}{r} \partial_{r}\left(\frac{r f^{\prime} f}{\left(1+f^{2}\right)^{2}}\left[\frac{n^{2}}{r^{2}}-\omega^{2}\right]\right)+\frac{1}{2}\right]=0 .
$$

Thus, as always, a solution is given by the vacuum value $f=0$ or by a solution of

$$
-\frac{1}{r} \partial_{r}\left(\frac{r f^{\prime} f}{\left(1+f^{2}\right)^{2}}\left[\frac{n^{2}}{r^{2}}-\omega^{2}\right]\right)+\frac{1}{2}=0 .
$$


Now we introduce the $g$ function

$$
1-g=\frac{1}{1+f^{2}}
$$

and get the equation

$$
-\frac{1}{r} \partial_{r}\left(r g^{\prime}\left[\frac{n^{2}}{r^{2}}-\omega^{2}\right]\right)+1=0
$$

We are looking for non-topological solutions of the $Q$-ball type. Hence, the relevant boundary conditions are: for a compacton starting at $r=0$

$$
g(r=0)=0, \quad g(r=R)=0, \quad g^{\prime}(r=R)=0
$$

or, for a shell-like compacton

$$
g\left(r=R_{i}\right)=0, \quad g^{\prime}\left(r=R_{i}\right)=0, \quad i=1,2, \quad R_{1}<R_{2} .
$$

The general solution of the e.o.m. is

$$
g^{\prime}(r)=\frac{1}{\frac{n^{2}}{r^{2}}-\omega^{2}}\left(\frac{r}{2}+A\right)
$$

and

$$
g(r)=-\frac{r^{2}}{4 \omega^{2}}-\frac{n^{2}}{4 \omega^{4}} \ln \left|n^{2}-\omega^{2} r^{2}\right|-\frac{A}{2 \omega^{2}} \ln \left|n^{2}-\omega^{2} r^{2}\right|+B .
$$

First of all we may exclude shell-like compactons, as from equation for $g^{\prime}$ we get that

$$
g^{\prime}(r=R)=0 \Rightarrow R^{2}=-2 A \text {. }
$$

Hence, there is only one positive $R$. Notice, $A$ must be negative. Further $g(0)=0$ gives

$$
B=\frac{n^{2}}{4 \omega^{4}} \ln \left|n^{2}\right|+\frac{A}{2 \omega^{2}} \ln \left|n^{2}\right|
$$

and

$$
g(r)=-\frac{r^{2}}{4 \omega^{2}}-\frac{n^{2}}{4 \omega^{4}} \ln \left|1-\frac{\omega^{2} r^{2}}{n^{2}}\right|-\frac{A}{2 \omega^{2}} \ln \left|1-\frac{\omega^{2} r^{2}}{n^{2}}\right| .
$$

The last constant $A$ can be found from $g(R)=0$

$$
0=-\frac{R^{2}}{4 \omega^{2}}-\frac{n^{2}}{4 \omega^{4}} \ln \left|1-\frac{\omega^{2} R^{2}}{n^{2}}\right|-\frac{A}{2 \omega^{2}} \ln \left|1-\frac{\omega^{2} R^{2}}{n^{2}}\right|
$$

But $R^{2}=-2 A$, thus (after multiplication by $4 \omega^{2} / n^{2}$

$$
0=\frac{2 A \omega^{2}}{n^{2}}-\ln \left|1+\frac{2 A \omega^{2}}{n^{2}}\right|-\frac{2 A \omega^{2}}{n^{2}} \ln \left|1+\frac{2 A \omega^{2}}{n^{2}}\right|
$$

or

$$
0=p-(1+p) \ln |1+p|,
$$

where $p=2 A \omega^{2} / n^{2}$. This equation has two solutions $p=0$ and $p=p_{0}<-1$. But in order to have a non-singular solution $g$, the radial coordinate cannot be larger than $r<n / w$. So, the size of the compacton must be also smaller than this critical value 
$R<n / w$. As we know $R=\sqrt{-2 A}$, where $2 A=p n^{2} / w^{2}$ if we use the definition of $p$. Then, $R=\sqrt{-p} n / w<n / w$. Thus finally, $0<\sqrt{-p}<1$ i.e., $p \in(-1,0)$, which is in contradiction with our previous observation that $p_{0}<-1$.

The generalization to other potentials is unfortunately difficult. We are left with a quite complicated second order nonlinear ODE for $g$. Fortunately, one may prove that for any old baby potential (6) there are no non-spinning $(n=0)$, non-topological $Q$-balls. Instead of equation (29), we get

$$
\frac{1}{r} \partial_{r}\left(r g^{\prime}\right)+g^{s-1}=0,
$$

where the radial coordinate has been properly re-scaled. Assuming a series expansion in the vicinity of the origin

$$
g(r)=A r^{a}+\ldots
$$

we get

$$
a=\frac{2}{2-s}, \quad a^{2}+A^{s-2}=0
$$

The second formula leads to a complex or negative value of $A$, which is unacceptable as $g$ must be positive. A similar effect occurs if we try an expansion at any finite $R$. Then we have

$$
a(a-1)+A^{s-2}=0 .
$$

We can get an acceptable $A$ if $0<a<1$. But this gives $s<0$, which is excluded for obvious reasons. Hence, no $n=0, Q$-balls are possible.

The non-existence of non-topological $Q$-balls in the pure baby Skyrme models is a rather new feature which has no analog in the full baby Skyrme model. On the contrary, in the full theory one expects to find non-topological time dependent solutions, compact or non-compact, depending on the chosen form of the potential [7]. Moreover, this result is also unexpected, as topological $Q$-balls have been found by Gisiger and Paranjape [9], with properties similar to $Q$-baby skyrmions. Apparently, the restricted model leads to non-trivial solutions which reflect properties of the full model only for field configurations with a non-trivial topological charge.

\section{Conclusions}

In this paper the pure baby Skyrme model has been analyzed. It was our main aim to investigate whether solutions of this simplified model may tell us something about the baby skyrmions, i.e., solitons in the full baby Skyrme theory. Such a correspondence between solutions of the two models is by no means obvious. Due to the huge amount of symmetries in the restricted model, one could rather expect a completely different behavior of solutions. We found, nevertheless, that many properties of baby skyrmions are sufficiently well described by solutions of the pure model. Moreover, as the restricted model is integrable in the $(2+0)$ dimensional case (in $(2+1)$ dimensions the model has the property of generalized integrability), we were able to perform all calculations analytically.

The main findings are as follows:

- Topological (charge) as well as geometrical (nucleus/shell) features of baby skyrmions are captured already by the soliton solutions of the pure model 
- Energies of baby skyrmions are reasonably approximated by the energies of the solitons of the pure model. The approximation becomes better and better while $\mu \rightarrow \infty$

- There is a coincidence between the existence of compact solitons in the pure baby model and the existence (for some values of the parameters) of multi-babyskyrmions in the full baby Skyrme model

- There are no topologically trivial $Q$-ball configurations

The first three results show that the static properties of baby skyrmions may be qualitatively and quantitatively described by solutions of the pure model. They indicate that the topological and geometrical properties are governed by the Skyrme term and the potential, while the quadratic part of the full baby Skyrme model only quantitatively modifies them. Especially, the third result can be potentially important as it gives a chance for an analytical treatment of the issue of the stability of multi skyrmions. So the purely quartic model, despite its high symmetry and probable integrability, shares some properties with the full model, and may probably be used to approximate the full model in some sense (for approximation of solitons by compactons in the case of a scalar field theory see [19]).

The fact that no topologically trivial $Q$-balls exist in the restricted model leads to a different conclusion. $Q$-balls in the full baby Skyrme model are not approximated by any solutions of the pure baby model but rather by solutions of the $C P^{1}$ model, i.e., another simplified version of the baby Skyrme theory, which consists of the quadratic part and potential. Of course, such a model does not allow for static solutions and, therefore, is useless in the context of the static baby skyrmions.

The identification of simplified models relevant for the study of properties of topological solitons (the pure baby Skyrme model) and non-topological solutions (the $C P^{1}$ model with potential) is another result of the paper.

\section{Acknowledgements}

C.A. and J.S.-G. thank MCyT (Spain), FEDER (FPA2005-01963) and Xunta de Galicia (grant INCITE09.296.035PR and Conselleria de Educacion) for financial support. A.W. acknowledges support from the Ministry of Science and Higher Education of Poland grant N N202 126735 (2008-2010). Further, A.W. thanks Ya. Shnir for discussion.

\section{References}

[1] T.H.R. Skyrme, Proc. Roy. Soc. Lon. 260, 127 (1961); Nucl. Phys. 31, 556 (1961); J. Math. Phys. 12, 1735 (1971).

[2] B.M.A.G. Piette, B.J. Schoers and W.J. Zakrzewski, Z. Phys. C 65 (1995) 165; B.M.A.G. Piette, B.J. Schoers and W.J. Zakrzewski, Nucl. Phys. B 439 (1995) 205.

[3] R.A. Leese, M. Peyrard and W.J. Zakrzewski Nonlinearity 3 (1990) 773; B.M.A.G. Piette and W.J. Zakrzewski, Chaos, Solitons and Fractals 5 (1995) 2495; P.M. Sutcliffe, Nonlinearity (1991) 41109. 
[4] T. Weidig, Nonlinearity 12 (1999) 1489.

[5] P. Eslami, M. Sarbishaei and W.J. Zakrzewski, Nonlinearity 13 (2000) 1867.

[6] M. Karliner, I. Hen, Nonlinearity 21 (2008) 399-408; M. Karliner, I. Hen, arXiv:0901.1489

[7] C. Adam, P. Klimas, J. Sanchez-Guillen, A. Wereszczynski, Phys. Rev. D 80, 105013 (2009).

[8] A.A. Belavin, A.M. Polyakov, JETP Lett. 22 (1975) 245; S.L. Soundhi, A. Karlhede, S.A. Kivelson, E.H. Rezayi, Phys. Rev. B 47 (1993) 16419; N.R. Walet, T. Weidig, Europhys. Lett. 55 (2001) 633.

[9] T. Gisiger, M.B. Paranjape, Phys. Rev. D 55 (1997) 7731.

[10] C. Adam, J. Sanchez-Guillen, A. Wereszczynski, J. Math. Phys. 47, 022303 (2006).

[11] A.N. Leznov, B.M.A.G. Piette, W.J. Zakrzewski, J. Math. Phys. 38 (1997) 3007.

[12] P. Rosenau, J. H. Hyman, Phys. Rev. Lett. 70 (1993) 564; D. Bazeia, L. Lozano, R. Menezes, Phys. Lett. B 668 (2008) 246.

[13] Arodź H, Acta Phys. Polon. B 33 (2002). 1241; Arodź H, Acta Phys. Polon. B 35 (2004) 625.

[14] O. Alvarez, L.A. Ferreira, J. Sanchez-Guillen, Nucl. Phys. B529 (1998) 689; Int. J. Mod. Phys. A 24, 1825 (2009).

[15] M. de Innocentis, R.S. Ward, Nonlinearity 14 (2001) 663; hep-th/0103046

[16] K. Arthur, G. Roche, D.H. Tchrakian, Y. Tang, J. Math. Phys. 37 (1996) 2569.

[17] C. Adam, J. Sanchez-Guillen, A. Wereszczynski, arXiv:1001.4544.

[18] H. Aratyn, L.A. Ferreira, A.H. Zimerman, Phys. Lett. B 456, 162 (1999).

[19] J. Lis, arXiv:0911.3423. 